\title{
Broadband GaAs MESFET and GaN HEMT Resistive Feedback Power Amplifiers
}

\author{
Karthikeyan Krishnamurthy, Ramakrishna Vetury, Stacia Keller, Umesh Mishra, Mark J. W. Rodwell, and \\ Stephen I. Long
}

\begin{abstract}
We report 0.2 to 6-GHz MMIC power amplifiers with 12-dB gain, over 23-dBm output power, and more than $25 \%$ power-added efficiency (PAE) in a GaAs MESFET technology offering 18-GHz $f_{\tau}$ and $12-\mathrm{V}$ breakdown. These circuits have gain-bandwidth products of $\sim 1.3 \cdot f_{\tau}$ and are more efficient than distributed power amplifiers. A first demonstration of similar circuits in GaN/AIGaN HEMT technology yielded 11-dB gain, 0.2 to $7.5-\mathrm{GHz}$ bandwidth amplifiers with over $31.5-\mathrm{dBm}$ output power and up to $15 \%$ PAE. With improved devices and models we expect significantly higher power from the GaN HEMT circuits.
\end{abstract}

Index Terms-Broadband amplifiers, FET circuits, power amplifiers.

\section{INTRODUCTION}

W IDE-BANDWIDTH power amplifiers are key components in phased-array radars and instrumentation. These amplifiers must operate over a decade bandwidth with high output power and power-added efficiency (PAE). Simple lumped broadband amplifiers have gain-bandwidth products limited by the current-gain cut-off frequency $f_{\tau}$. Distributed or traveling-wave amplifiers (TWAs) can provide gain-bandwidth products up to the power-gain cutoff frequency, $f_{\max }[1],[2]$. Conventional distributed amplifiers with reverse termination have poor efficiency due to the drain-line reverse wave. Reported monolithic distributed power amplifiers typically have $10 \%$ to $15 \%$ PAE [3], [4]. Distributed amplifiers using tapered impedance drain-lines can in theory provide efficiencies up to the class-A limit of $50 \%$, by eliminating the reverse termination [5], [6]. Tapered drain-line TWAs require high impedance transmission lines with limited current carrying capability, and thus are hard to realize for high output powers in monolithic form. Cascode-delay-matched distributed amplifiers have also been shown to be an efficient topology realizable for high output powers [7]. Though these modifications improve the efficiency, distributed amplifiers in general occupy a large die area.

We had presented [8] an alternative lumped broadband power amplifier based on the $f_{\tau}$-doubler topology [9]. The $f_{\tau}$-doubler small-signal amplifiers have shown gain-bandwidth products

Manuscript received January 18, 2000; revised March 27, 2000. This work was supported by the Office of Naval Research / IMPACT MURI under Contract N000 14-96-1-1215.

K. Krishnamurthy was with the Department of Electrical and Computer Engineering, University of California, Santa Barbara, CA 93106, USA. He is now with Gtran, Inc., Westlake, CA 91362 USA (e-mail: karthik@gtran.com).

R. Vetury, S. Keller, U. Mishra, M. J. W. Rodwell, and S. I. Long are with the Department of Electrical and Computer Engineering, University of California, Santa Barbara, CA 93106, USA.

Publisher Item Identifier S 0018-9200(00)05914-X.

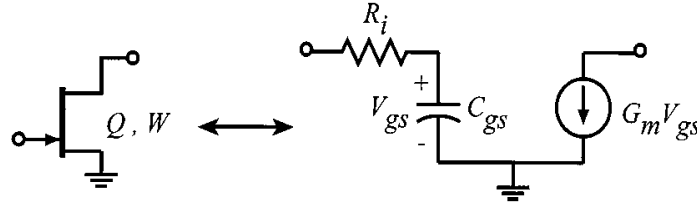

Fig. 1. Simplified ac FET model used for analysis.

approaching twice the transistor $f_{\tau}$ [10], [11]. As a power amplifier $f_{\tau}$-doubler circuits can provide higher efficiency and smaller die area than conventional distributed amplifiers. In this paper we report GaAs MESFET power amplifiers with 12-dB gain and bandwidths as high as $33 \%$ of $f_{\tau}$, comparable to distributed power amplifiers. PAE in excess of $25 \%$ is obtained, higher than reported for distributed amplifiers. AlGaN/GaN HEMTs have high $f_{\tau} V_{\text {br }}$ products [12], and are good candidates for high-power broadband amplifiers. First results on $f_{\tau}$-doubler power amplifiers using single and dual-gate $\mathrm{GaN}$ HEMTs are also presented [13].

\section{CiRCUIT DESIGN}

As impedance transformation over a decade bandwidth is difficult to realize on-wafer, most wideband amplifiers use $50-\Omega$ output loading. Transistor output power is then limited to

$$
P_{\text {out }} \leq \frac{\left(V_{\mathrm{br}}-V_{k}\right)^{2}}{8 Z_{\circ}}
$$

where $V_{\mathrm{br}}$ is the breakdown voltage, $V_{k}$ the knee voltage and $Z_{o}=50 \Omega$. The device periphery $W$ is chosen to provide saturation current $I_{\mathrm{DSS}}$, determined by the load-line constraint

$$
I_{\mathrm{DSS}}=\frac{\left(V_{\mathrm{br}}-V_{k}\right)}{Z_{o}} .
$$

A common-source amplifier, using a simplified FET model of Fig. 1, has a short circuit current gain

$$
H_{21, \mathrm{cs}}=\left(\frac{f_{\tau}}{j f}\right)
$$

reaching unity at $f_{\tau}$ (Fig. 2). Capacitive degeneration [3] provides higher bandwidth at lower gain with constant gain-bandwidth product. To obtain sufficiently high PAE (up to $40 \%$ ), gains $>10 \mathrm{~dB}$ are required, limiting bandwidth to $f_{3 \mathrm{~dB}}<f_{\tau} / 3$. With other parasitics considered, bandwidth falls significantly below $f_{\tau} / 3$.

Darlington amplifiers have higher bandwidth than common-source amplifiers but provide lesser efficiency. 


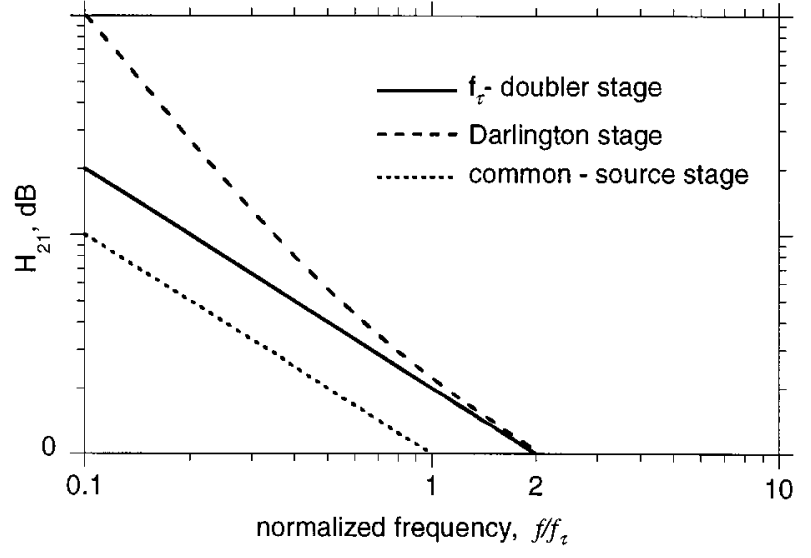

Fig. 2. Short circuit current gain $H_{21}$ for common-source, Darlington, and $f_{\tau}$-doubler configurations

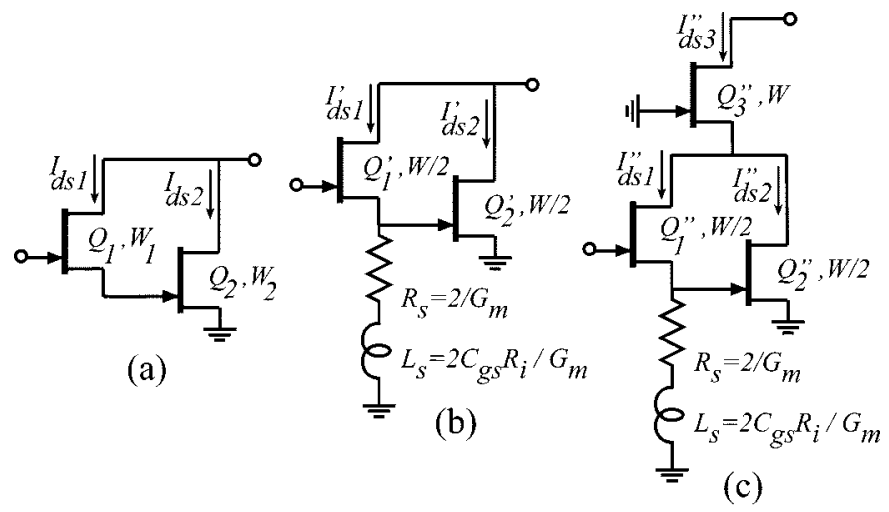

Fig. 3. AC schematics. (a) Basic Darlington. (b) $f_{\tau}$-doubler configuration. (c) Cascode $f_{\tau}$-doubler configuration.

If the drain of the common-drain device is connected to the amplifier output [Fig. 3(a)], then the current gain

$$
H_{21, \text { Darlington }}=\frac{\left(1+j 2 f / f_{\tau}\right)}{\left(j f / f_{\tau}\right)^{2}}
$$

is twice that of a common-source stage at high frequencies (Fig. 2), reaching unity at $2 f_{\tau}$. The peak output power obtainable from a Darlington stage is

$$
P_{\text {out,Darlington }} \leq \frac{\left(V_{\mathrm{br}}-V_{k}-V_{p}\right)^{2}}{8 Z_{o}} \equiv \frac{V_{o}^{2}, \mathrm{p}-\mathrm{p}}{8 Z_{o}}
$$

which is slightly less than the common-source case [see (1)] because the peak-peak output voltage $V_{o, \mathrm{p}-\mathrm{p}}$ is now reduced by the pinch-off voltage $V_{p}$. Of greater importance for efficiency is the ratio of the ac currents of the two devices. This ratio is independent of device sizing and is frequency-dependent, given by

$$
\frac{I_{\mathrm{ds} 1}}{I_{\mathrm{ds} 2}}=\frac{\left(j f / f_{\tau}\right)}{\left(1+j f / f_{\tau}\right)} .
$$

At low frequencies, transistor $Q_{2}$ provides the entire ac output current. Thus, a Darlington amplifier designed for the same power level as the common-source power amplifier must have $I_{\mathrm{DSS} 2}=V_{o, \mathrm{p}-\mathrm{p}} / Z_{o}$ or $W_{2}=W$, the same periphery as the device in the common-source case. At high frequencies (of the order of $f_{\tau}$ ) the ac output current is provided equally by $Q_{1}$ and

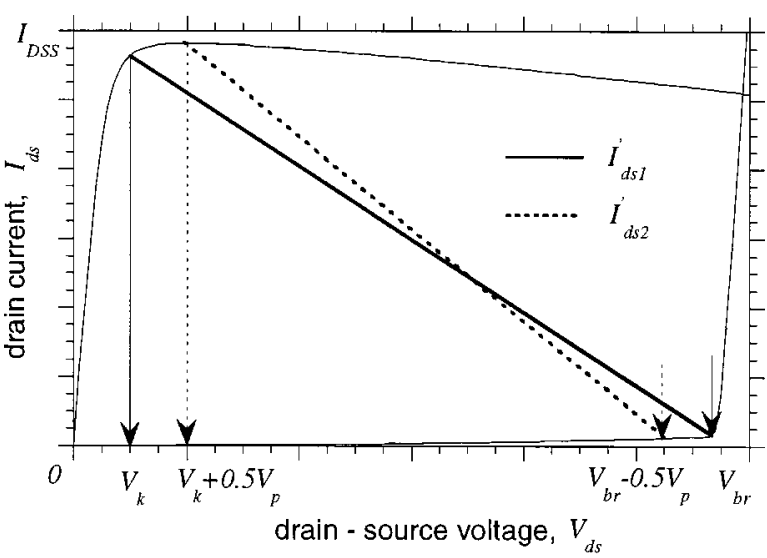

Fig. 4. Load-lines seen by the two devices in a $f_{\tau}$-doubler power amplifier, superimposed on the device $I_{\mathrm{ds}}-V_{\mathrm{ds}}$ plot.

$Q_{2}$. Thus, we must have $I_{\mathrm{DSS} 1}=V_{o, \mathrm{p}-\mathrm{p}} / 2 Z_{o}$ or $W_{1}=W / 2$. Therefore, in the Darlington power amplifier, the total device periphery and the net bias current are a factor of 1.5 higher than in a common-source power amplifier designed for the same ac output current. DC power consumption is a factor of 1.5 higher and the PAE is impaired. Improved bandwidth is obtained at the cost of lower PAE. Potential instability due to negative input resistance

$$
\begin{aligned}
Z_{\mathrm{in}, \text { Darlington }}= & R_{i 1}+R_{i 2}-\frac{G_{m 1}}{\omega^{2} C_{\mathrm{gs} 1} C_{\mathrm{gs} 2}} \\
& +\frac{1}{j \omega}\left(\frac{1+G_{m 1} R_{i 2}}{C_{\mathrm{gs} 1}}+\frac{1}{C_{\mathrm{gs} 2}}\right)
\end{aligned}
$$

is a further limitation of Darlingtons.

The $f_{\tau}$-doubler power amplifier [Fig. 3(b)] is a modified Darlington stage where suitable source loading of $Q_{1}^{\prime}$ splits the input voltage equally between $Q_{1}^{\prime}$ and $Q_{2}^{\prime}$, so that $I_{\mathrm{ds} 1}^{\prime}=I_{\mathrm{ds} 2}^{\prime}$ at all frequencies. The source loading consists of a resistor $R_{s}=$ $2 / G_{m}$ in series with an inductor $L_{s}=2 C_{\mathrm{gs}} R_{i} / G_{m}$. Published $f_{\tau}$-doubler amplifiers [10], [11] have not incorporated this inductor. $L_{s}$ is required to obtain equal input voltage division between $Q_{1}^{\prime}$ and $Q_{2}^{\prime}$ for frequencies $f \sim 1 / 2 \pi R_{i} C_{\mathrm{gs}}$; the $L_{s} / R_{s}$ time constant being equal to the $R_{i} C_{\mathrm{gs}}$ time constant.

With this source loading, $Q_{1}^{\prime}$ and $Q_{2}^{\prime}$ carry equal ac drain current for all frequencies. Thus, for the two devices to reach saturation simultaneously, we require that $I_{\mathrm{DSS} 1}^{\prime}=I_{\mathrm{DSS} 2}^{\prime}=$ $V_{o, \mathrm{p}-\mathrm{p}} / 2 Z_{o}$. Hence the two devices should have half the periphery of a common-source stage of equal output power (i.e., $W / 2$ ). The two devices $Q_{1}^{\prime}$ and $Q_{2}^{\prime}$ have marginally different load-lines given by $\left(2 Z_{o}+2 / G_{m}\right)$ and $2 Z_{o}$, respectively. This is due to the extra source loading seen by $Q_{1}^{\prime}$, which limits the peak-peak $V_{\mathrm{ds}}$ of $Q_{1}^{\prime}$ and $Q_{2}^{\prime}$ to $\left(V_{\mathrm{br}}-V_{k}\right)$ and $\left(V_{\mathrm{br}}-V_{k}-V_{p}\right)$, respectively (Fig. 4). This does not significantly affect the output power, provided that $Z_{o} \gg 1 / G_{m}$ or $\left(V_{\mathrm{br}}-V_{k}\right) \gg V_{p}$. As in the case of the Darlington amplifier, the peak output power

$$
P_{\mathrm{out}, f_{\tau}-\text { doubler }} \leq \frac{\left(V_{\mathrm{br}}-V_{k}-V_{p}\right)\left(V_{\mathrm{br}}-V_{k}-V_{p} / 2\right)}{8 Z_{o}}
$$

is slightly smaller than the common-source power amplifier. DC power consumption is similar to the common-source case provided that dc power dissipation in the source resistance is elim- 


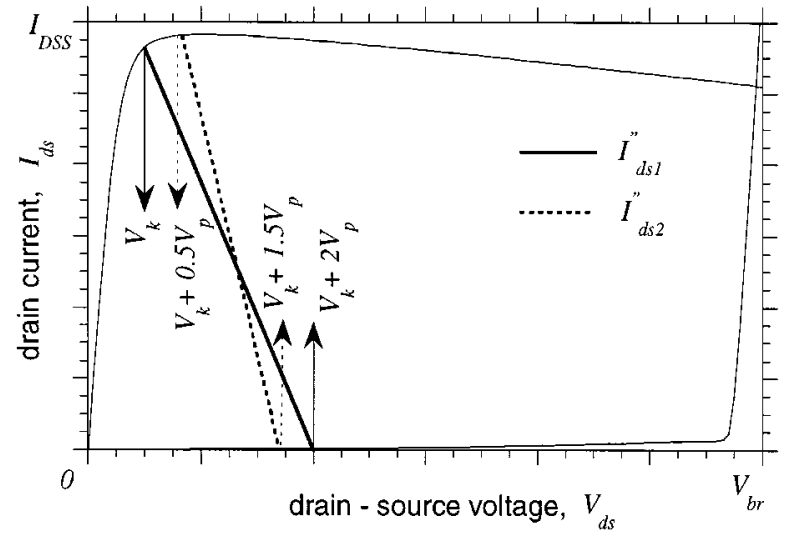

Fig. 5. Load-lines seen by the two devices, $Q_{1}^{\prime \prime}$ and $Q_{2}^{\prime \prime}$ in a cascode $f_{\tau}$-doubler power amplifier, superimposed on the device $I_{\mathrm{ds}}-V_{\mathrm{ds}}$ plot.

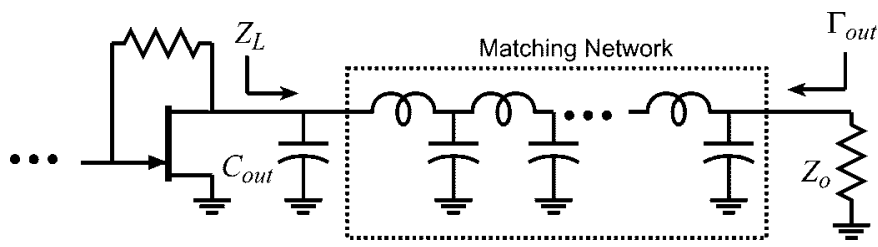

Fig. 6. $\pi$-section output matching networks for broadband power amplifiers.

inated by grounding the source of $Q_{1}^{\prime}$ for dc using a RF choke. The current gain of the $f_{\tau}$-doubler stage

$$
H_{21, f_{\tau}-\text { doubler }}=\left(\frac{2 f_{\tau}}{j f}\right)
$$

has a single pole and is unity at $2 f_{\tau}$ (Fig. 2). Thus bandwidth is improved without significant loss in efficiency. Stability is also improved as the input impedance of the $f_{\tau}$-doubler

$$
Z_{\mathrm{in}, f_{\tau}-\text { doubler }}=R_{i 1}+R_{i 2}+\frac{1}{j \omega}\left(\frac{1}{C_{\mathrm{gs} 1}}+\frac{1}{C_{\mathrm{gs} 2}}\right)
$$

no longer has a negative resistance.

Because the $f_{\tau}$-doubler topology alleviates the bandwidth limitation due to $C_{\mathrm{gs}}$, the parasitic $C_{\mathrm{gd}}$ and $C_{\mathrm{ds}}$ may then become significant. Addition of a common-gate stage to form a cascode $f_{\tau}$-doubler [Fig. 3(c)] reduces the effect of $C_{\mathrm{gd}}$ substantially. In this case, since the $f_{\tau}$-doubler is driving a low input impedance common-gate stage, the two devices $Q_{1}^{\prime \prime}$ and $Q_{2}^{\prime \prime}$ drive widely different loads of $4 / G_{m}$ and $2 / G_{m}$, respectively (Fig. 5). However the power dissipated in $Q_{1}^{\prime \prime}$ and $Q_{2}^{\prime \prime}$ is a small fraction of the net output power as in the case of a cascode (common-source - common-gate) amplifier.

For all the configurations considered above, the capacitances $C_{\mathrm{gd}}$ and $C_{\mathrm{ds}}$ load the amplifier output. Broadband $\pi$-sections (Fig. 6) could be used to partially absorb the net output capacitance $\left(C_{\text {out }}\right)$ and improve matching. In small signal amplifiers, these networks are designed to minimize the magnitude of the output reflection coefficient $\left(\Gamma_{\text {out }}\right)$, over the bandwidth.

In power amplifiers there is an additional constraint on the matching network, to improve output power and PAE at the upper band edge. In the absence of the matching network, the load-line deviates from $Z_{o}$ with increasing frequency (Fig. 7) due to $C_{\text {out }}$. With the matching network (Fig. 6), the peak output

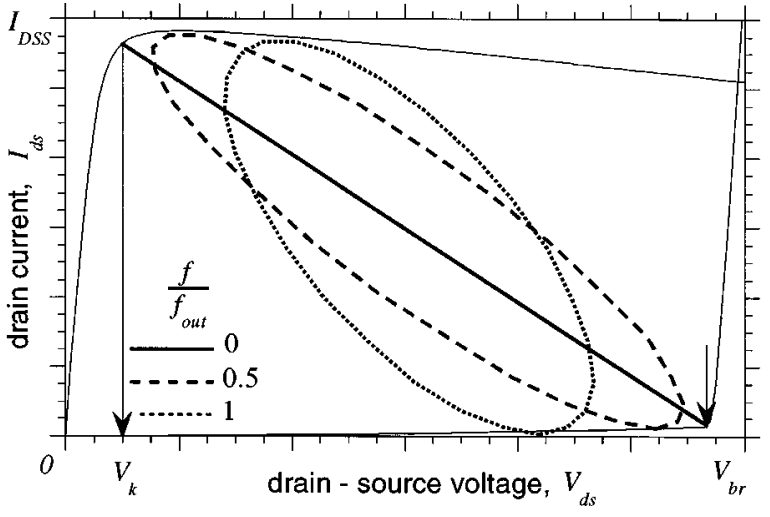

Fig. 7. Variation of load-line with frequency due to the presence of $C_{\text {out }}$ $\left(f_{\text {out }}=I_{\mathrm{DSS}} / 2 \pi C_{\text {out }} V_{\mathrm{br}}\right)$.

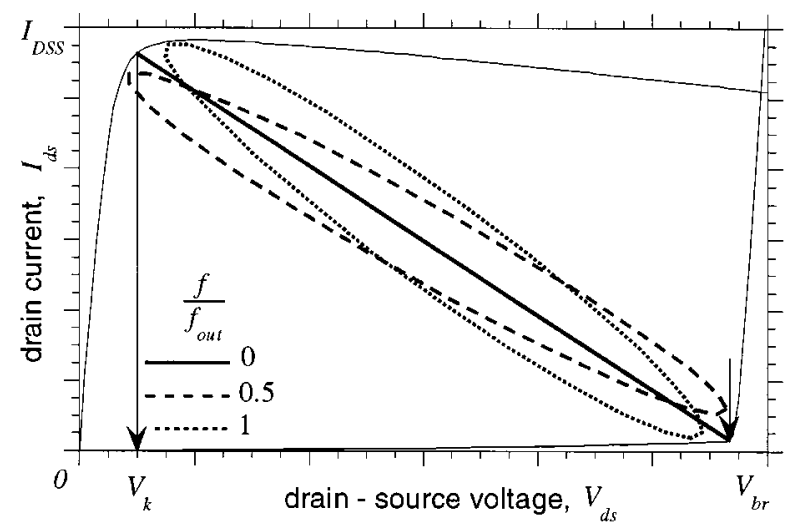

Fig. 8. Variation of load-line with frequency in the presence of a four-section matching network at the output.

power as a function of frequency is dependent on $\operatorname{Re}\left(Z_{L}\right)$ or $\operatorname{Re}\left(Y_{L}\right)$ as decided by the magnitude of $Z_{L}$ [14]. For the case of $\left|Z_{L}\right|<Z_{o}$, the load-line is limited by the maximum allowed current swing $\left(I_{\mathrm{DSS}}\right)$. The peak output power $P_{\text {out }}(f)$ is now less than the optimum case $\left[P_{\text {out }}\right.$ in (1)] and given by

$$
\frac{P_{\text {out }}(f)}{P_{\text {out }}}=\frac{\operatorname{Re}\left(Z_{L}\right)}{Z_{o}} .
$$

For the case of $\left|Z_{L}\right|>Z_{O}$, the load-line is limited by the maximum allowed voltage swing $\left(V_{\mathrm{br}}\right)$ and the peak output power is now

$$
\frac{P_{\text {out }}(f)}{P_{\text {out }}}=\operatorname{Re}\left(Y_{L}\right) Z_{\circ} .
$$

So to obtain the maximum output power, the matching networks have to be designed to minimize $\operatorname{Im}\left(Z_{L}\right)$ and $\operatorname{Im}\left(Y_{L}\right)$ over the bandwidth. Fig. 8 shows the load-line variation in the presence of a four-section matching network. These networks will improve PAE over a bandwidth of at most $\Delta f_{\text {out }}=I_{\mathrm{DSS}} / 2 \pi C_{\mathrm{out}} V_{\mathrm{br}}$, the output pole frequency.

\section{EXPERIMENTAL RESULTS}

\section{A. GaAs MESFET Power Amplifiers}

The ICs were fabricated using the TriQuint Semiconductor, Inc., TQTRx process. The circuits were simulated using TOM2 large signal MESFET models. Fig. 9 shows the circuit diagram 


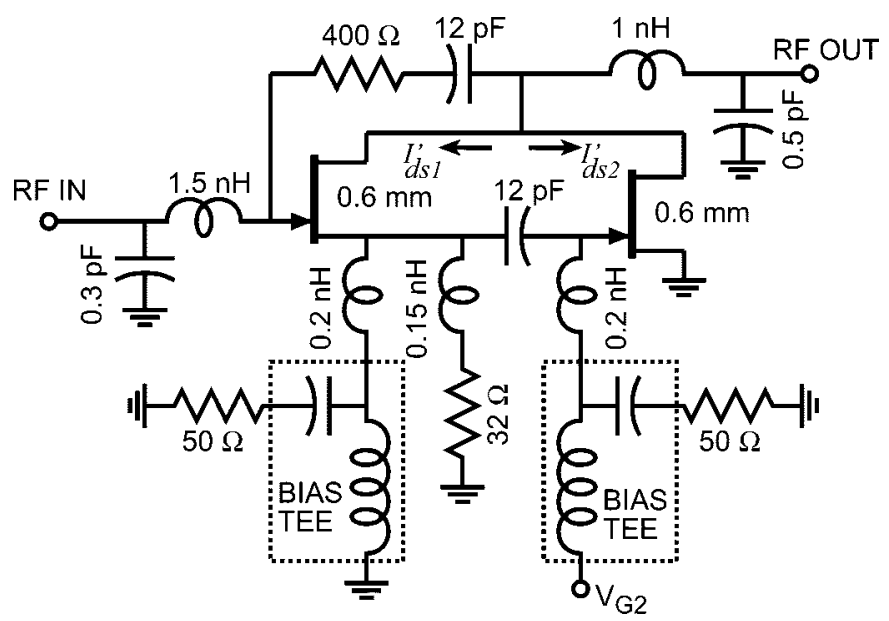

Fig. 9. Complete schematic of the GaAs MESFET $f_{\tau}$-doubler resistive feedback power amplifier.

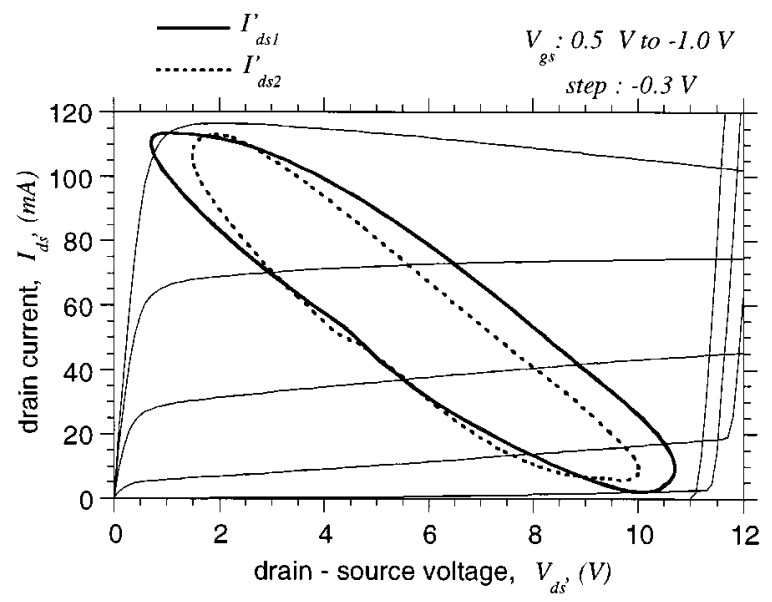

Fig. 10. Simulated load-lines of the devices in the GaAs MESFET $f_{\tau}$-doubler resistive feedback power amplifier (frequency $=3 \mathrm{GHz}$ ), superimposed on the simulated device $I_{\mathrm{ds}}-V_{\mathrm{ds}}$ plot.

of the $f_{\tau}$-doubler power amplifier. Resistive feedback matches input and output impedances to $50 \Omega$ without significant loss of efficiency. Broadband $\pi$-sections at the input and output were designed to improve matching. High $Q$ spiral inductors were obtained by using two layers of metallization. External bias tees were used to independently bias the two devices. The source of the first device was grounded for dc through a bias tee. This also facilitates monitoring the device bias currents. The source loading was split into three parallel sections, with the bias tee's $50-\Omega$ ac ports providing part of the RF termination. This helps in monitoring the RF current split between the two devices. For maximum efficiency operation, bias is adjusted to fill the load-line of each device. The simulated load-line for the two devices (Fig. 10) at $3 \mathrm{GHz}$ shows a nearly equal current division. The capacitive current seen in the load-line is due to the device parasitic capacitance at the drain. Measured power performance (output power, transducer power gain, and PAE) at 5 $\mathrm{GHz}$ (Fig. 11) shows a peak output power of $23 \mathrm{dBm}$ with $27 \%$ PAE.

Fig. 12 shows the circuit diagram of the cascode $f_{\tau}$-doubler power amplifier. A secondary $R C$ feedback network, effective at high frequency, provides unconditional stability. Fig. 13

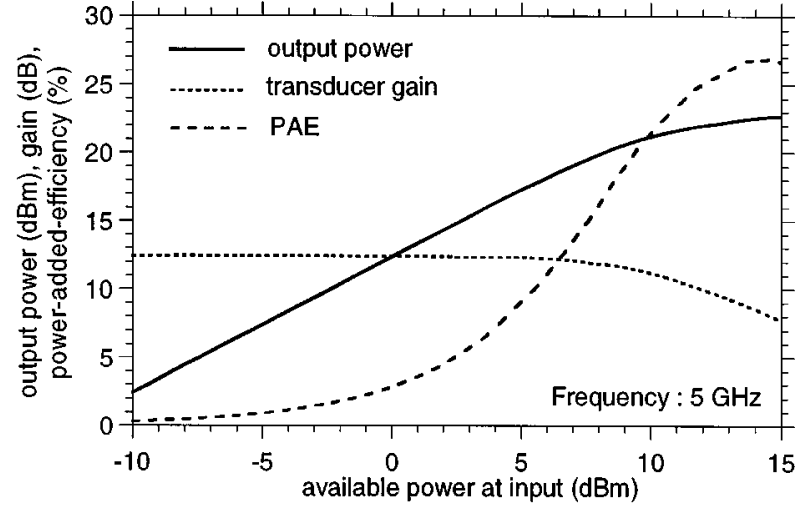

Fig. 11. Power performance of the GaAs MESFET $f_{\tau}$-doubler power amplifier at $5 \mathrm{GHz}$.

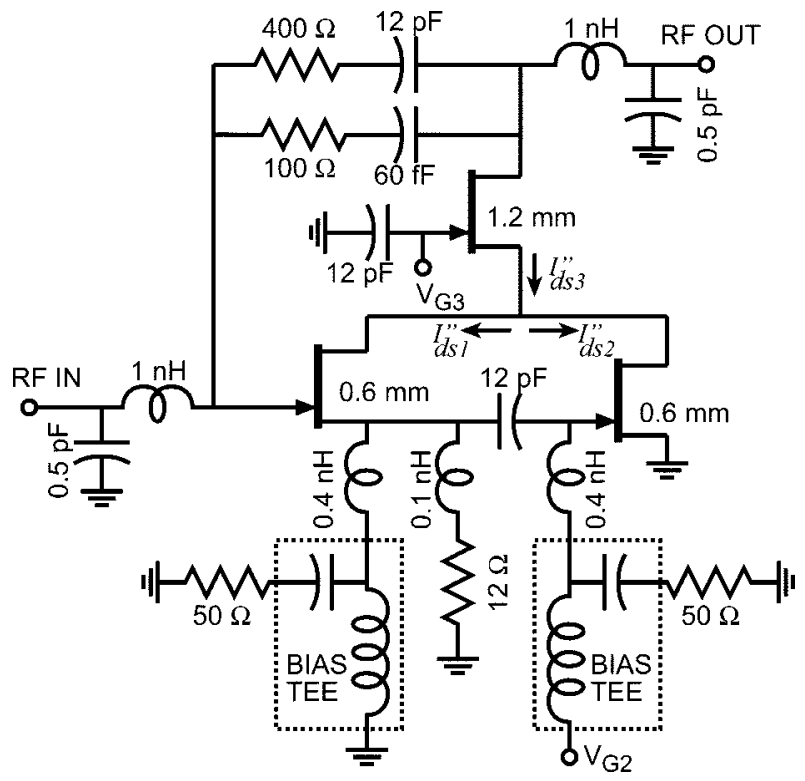

Fig. 12. Complete schematic of the GaAs MESFET cascode $f_{\tau}$-doubler resistive feedback power amplifier.

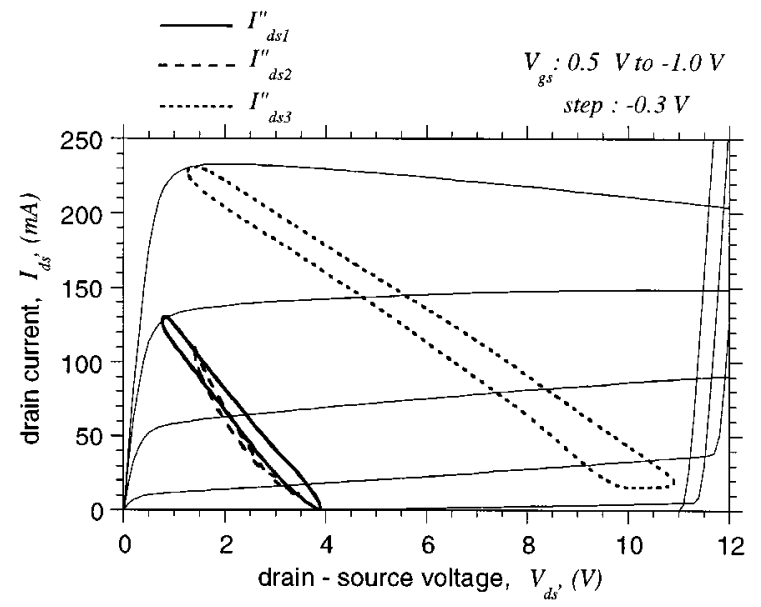

Fig. 13. Simulated load-lines of the devices in the GaAs MESFET cascode $f_{\tau}$-doubler resistive feedback power amplifier (frequency $=3 \mathrm{GHz}$ ), superimposed on the simulated device $I_{\mathrm{ds}}-V_{\mathrm{ds}}$ plot.

shows the simulated load-line for the three devices at $3 \mathrm{GHz}$. Measured power performance at $6 \mathrm{GHz}$ (Fig. 14) shows a peak output power of $23 \mathrm{dBm}$ with $26 \%$ PAE. 


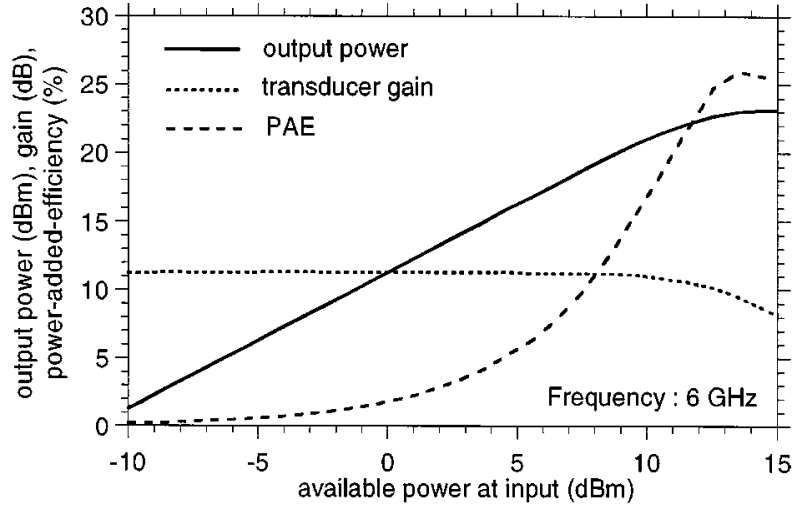

Fig. 14. Power performance of the GaAs MESFET cascode $f_{\tau}$-doubler power amplifier at $6 \mathrm{GHz}$.

(a)

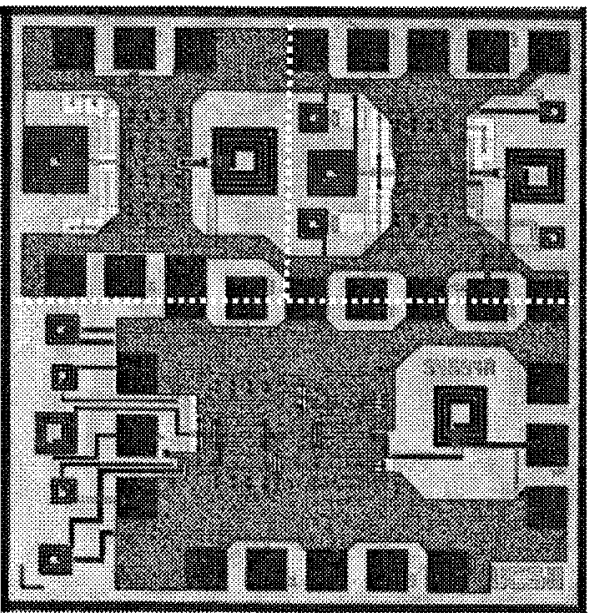

(c)

Fig. 15. Chip photograph of the GaAs MESFET. (a) Common-source. (b) $f_{\tau}$-doubler. (c) Cascode $f_{\tau}$-doubler power amplifier

Common-source power amplifiers were also fabricated for comparison. The die areas (Fig. 15) for the common-source, $f_{\tau}$-doubler, and cascode $f_{\tau}$-doubler amplifiers are $0.64 \mathrm{~mm}$ $\times 0.66 \mathrm{~mm}, 0.7 \mathrm{~mm} \times 0.7 \mathrm{~mm}$, and $1.36 \mathrm{~mm} \times 0.75 \mathrm{~mm}$, respectively. The $f_{\tau}$-doubler occupies similar die area as the common-source power amplifiers. $f_{\tau}$-doubler power amplifiers using dual-gate FETs should provide similar performance as cascode $f_{\tau}$-doubler power amplifiers without the excess die area.

Figs. 16-18 compare the power performance of the three circuits measured over 0.1 to $8 \mathrm{GHz}$. The common-source power amplifier provides $>24-\mathrm{dBm}$ output power over $2-\mathrm{GHz}$ bandwidth with $>30 \%$ PAE. The $f_{\tau}$-doubler power amplifier and the cascode $f_{\tau}$-doubler power amplifier provide $>23$-dBm output power over 0.2 to $5-\mathrm{GHz}$ and 0.2 to $6-\mathrm{GHz}$ bandwidth, respectively, with $>25 \%$ PAE. The roll-off in the output power and PAE is attributed to the FET output capacitance of $0.27 \mathrm{pF} / \mathrm{mm}$ resulting in an output pole at $\sim 10 \mathrm{GHz}$. Circuits with multisection $L C$ networks at the output, as discussed in the previous section, should provide improved PAE at the upper band-edge.

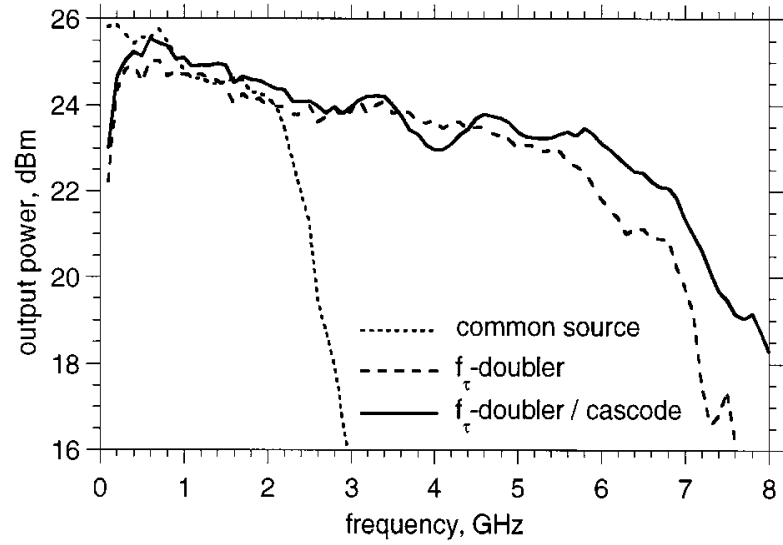

Fig. 16. Measured output power of the GaAs MESFET amplifiers as a function of frequency.

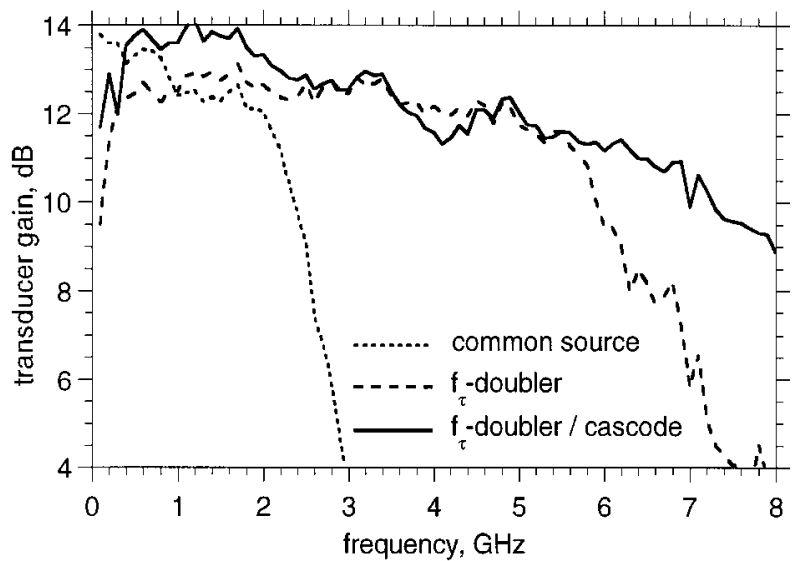

Fig. 17. Measured transducer gain of the GaAs MESFET amplifiers as a function of frequency.

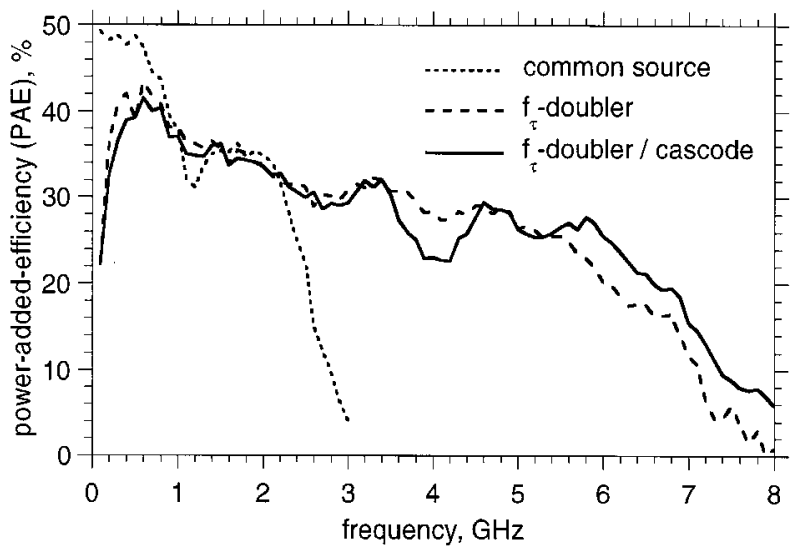

Fig. 18. Measured PAE of the GaAs MESFET amplifiers as a function of frequency.

\section{B. GaN HEMT Power Amplifiers}

GaN HEMTs with $0.7-\mu \mathrm{m}$ gate-length were fabricated on epitaxial material grown by MOCVD on $C$-plane sapphire. The layer structure and process details are discussed in [15]. A total device periphery of $W=2.4 \mathrm{~mm}$ was used to drive up to 1-A current into a $50-\Omega$ load, with the device breakdown exceeding $50 \mathrm{~V}$. Simulations predicted 10 -dB gain and 0.2 to $10-\mathrm{GHz}$ bandwidth. The devices were flip-chip bonded 


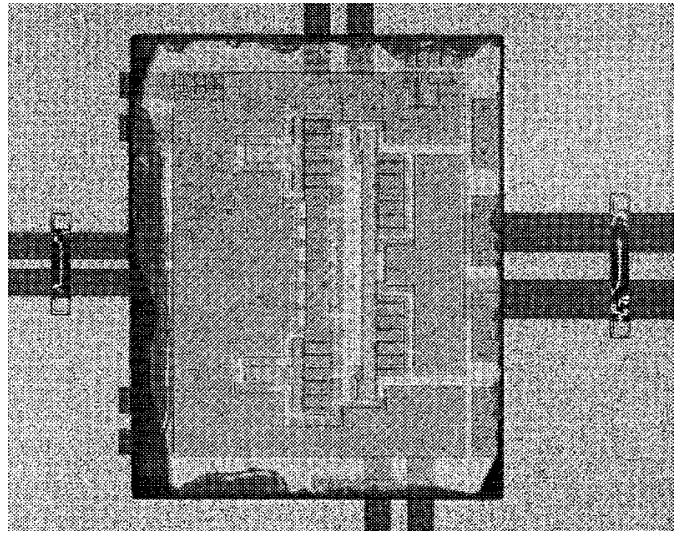

Fig. 19. Photograph of the GaN HEMT $f_{\tau}$-doubler resistive feedback power amplifier (GaN HEMT on transparent sapphire substrate, flip-chip bonded on AlN substrate).

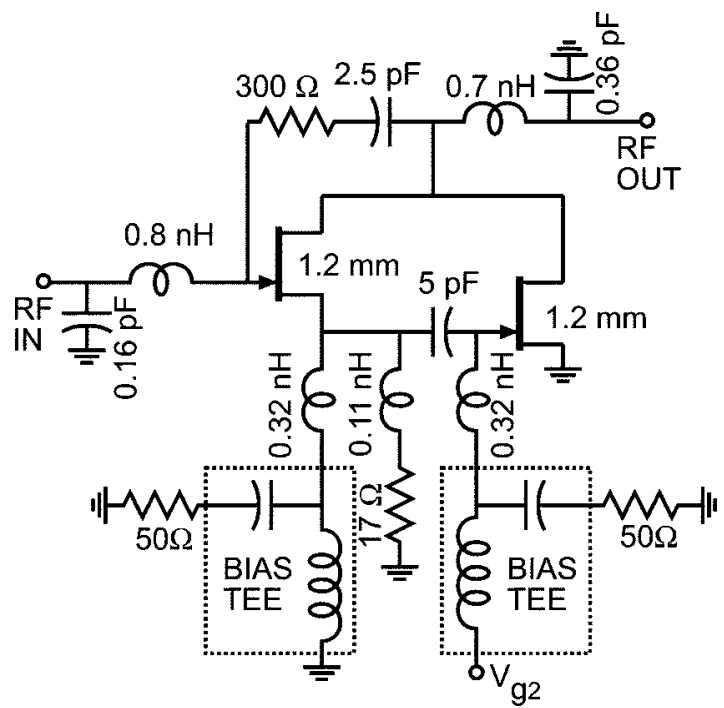

Fig. 20. Complete schematic of the GaN HEMT $f_{\tau}$-doubler resistive feedback power amplifier.

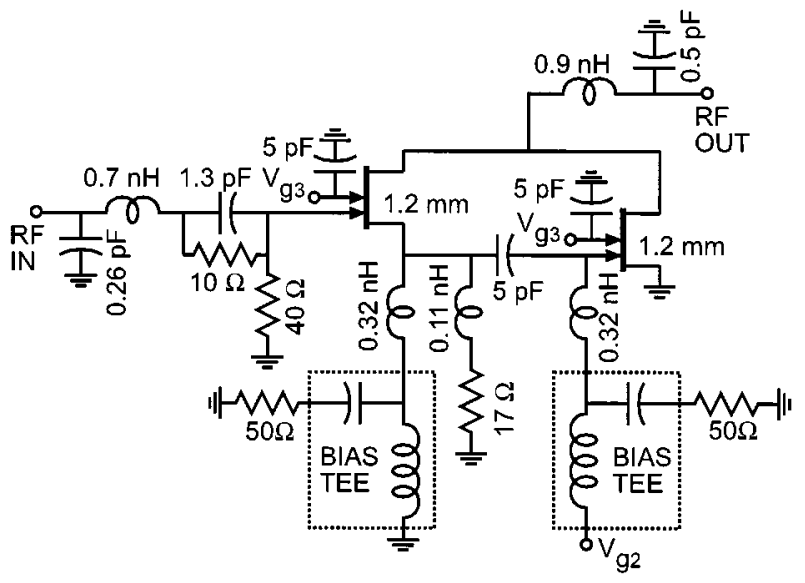

Fig. 21. Complete schematic of the GaN HEMT dual-gate cascode $f_{\tau}$-doubler resistive feedback power amplifier.

to AlN substrates (Fig. 19) which has the passive components ( $\mathrm{NiCr}$ resistors, $\mathrm{Si}_{3} \mathrm{~N}_{4}$ capacitors) and coplanar waveguide interconnects, and provides a low-resistance thermal path for

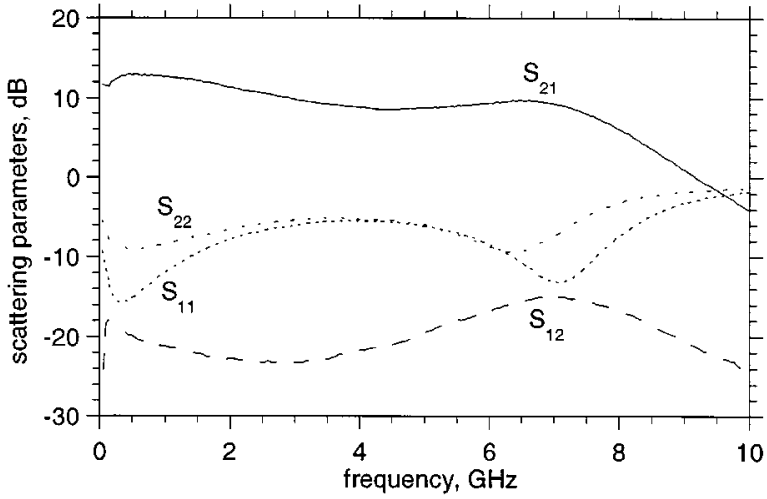

Fig. 22. Small-signal performance of the GaN HEMT $f_{\tau}$-doubler resistive feedback power amplifier.

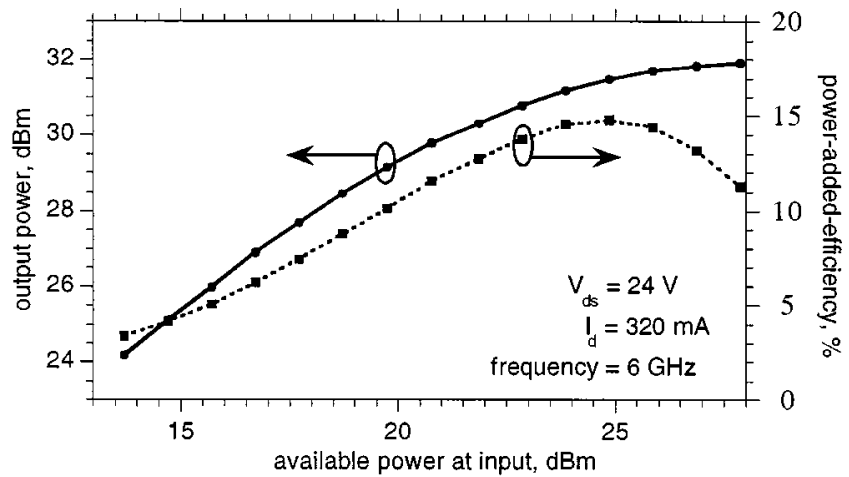

Fig. 23. Power performance of the GaN HEMT dual-gate cascode $f_{\tau}$-doubler power amplifier at $6 \mathrm{GHz}\left(V_{\mathrm{cs}}=24 \mathrm{~V}, I_{d}=320 \mathrm{~mA}\right)$.

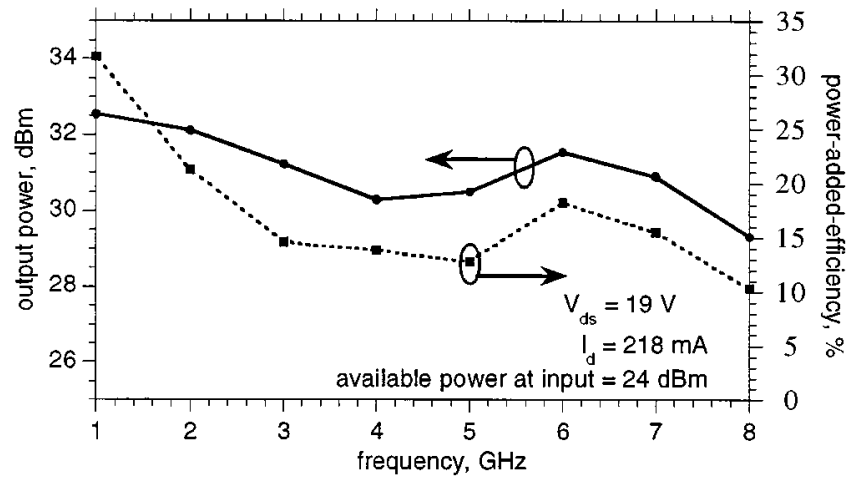

Fig. 24. Power performance of the GaN HEMT $f_{\tau}$-doubler resistive feedback power amplifier from 1 to $8 \mathrm{GHz}\left(V_{\mathrm{ds}}=19 \mathrm{~V}, I_{d}=218 \mathrm{~mA}\right.$, available power at input $=24 \mathrm{dBm}$ ).

efficient heat-sinking. The GaN die also provides a second plane of wiring for crossovers.

The single gate $f_{\tau}$-doubler power amplifier (Fig. 20) uses resistive feedback to match the input and output to $50 \Omega$. The dual-gate cascode $f_{\tau}$-doubler power amplifier (Fig. 21) uses capacitive division [3] to decrease gain and improve bandwidth. This design uses a resistive divider as a lossy input matching network. The divider also provides a low-impedance path for the gate bias preventing bias shifts at high RF drive. Biasing and $\pi$-section matching networks were done similar to the GaAs MESFET designs. The GaN die size is $1.1 \mathrm{~mm} \times 1.45 \mathrm{~mm}$ for either circuit. 
Measured small-signal scattering parameters of the resistive feedback power amplifiers (Fig. 22) shows $\sim 11-\mathrm{dB}$ small-signal gain with 0.2 to $7.5-\mathrm{GHz}$ bandwidth. The dual-gate cascode power amplifier provides $\sim 10-\mathrm{dB}$ small-signal gain with 0.2 to $8-\mathrm{GHz}$ bandwidth and up to $1.5-\mathrm{W}$ output power with 15\% PAE at $6 \mathrm{GHz}$ (Fig. 23). The resistive feedback power amplifier (Fig. 24) provides $>1$-W output power over 1 to $7 \mathrm{GHz}$ with $>10 \%$ PAE. The bandwidth is limited by the large extrinsic drain-source capacitance $C_{\mathrm{ds}}$ resulting from the flip-chip bonding. Improved transistor pad layouts should reduce the capacitance by a factor of two and improve circuit bandwidth. In both circuits the output power is observed to saturate at power levels well below what was expected from the transistor dc characteristics. Output waveform measurements at $2 \mathrm{GHz}$ with an oscilloscope indicate strong dc-RF dispersion in the HEMT I-V characteristics, possibly due to the presence of traps in the material. $V_{\text {Dsat }}$ increases and $I_{\text {DSS }}$ decreases when the device is operated at high frequencies. Also, leakage prevented complete cut-off of the channel current at high drain voltages.

\section{CONCLUSION}

The $f_{\tau}$-doubler topology is an alternative to the distributed amplifiers in realizing wideband power amplifiers with better efficiency in a smaller die area. An experimental circuit in a GaAs MESFET technology offering $18-\mathrm{GHz} f_{\tau}$ and $12-\mathrm{V}$ breakdown has achieved 0.2 to $6-\mathrm{GHz}$ bandwidth, $\sim 12-\mathrm{dB}$ gain, $>23-\mathrm{dBm}$ output power and $25 \%$ PAE. The bandwidth is $33 \%$ of $f_{\tau}$ and the gain-bandwidth product is $1.3 \cdot f_{\tau}$. Similar circuits using GaN HEMTs have yielded $\sim 11-\mathrm{dB}$ gain, 0.2 to $7.5-\mathrm{GHz}$ bandwidth, $\sim 31.5-\mathrm{dBm}$ output power, and up to $15 \%$ PAE. Circuits fabricated on improved material with reduced dc-RF dispersion have recently obtained up to $5-\mathrm{W}$ output power over a similar bandwidth, and will be reported subsequently. Better physical layout of the flip-chip bonded devices will reduce $C_{\mathrm{ds}}$ and increase bandwidth and efficiency. The efficiency at higher frequencies can be increased further by using improved broadband output networks.

\section{ACKNOWLEDGMENT}

The authors wish to thank R. Hickey of Triquint Semiconductor, Inc., and Dr. Y.-F. Wu of Nitres, Inc., for their technical support, without which this work would not have been possible. The authors also acknowledge the generous support of Dr. S. Taylor of TriQuint Semiconductor, Inc., in MESFET circuit fabrication.

\section{REFERENCES}

[1] B. Agarwal, A. E. Schmitz, J. J. Brown, M. Matloubian, M. G. Case, M. Le, M. Lui, and M. J. W. Rodwell, "112-GHz, 157-GHz, and 180-GHz InP HEMT traveling-wave amplifiers," IEEE Trans. Microwave Theory Tech., pp. 2553-2559, Dec. 1998.

[2] E. L. Ginzton, W. R. Hewlett, J. H. Jasberg, and J. D. Noe, "Distributed amplification," in Proc. I.R.E., vol. 36, 1948, pp. 956-969.

[3] Y. Ayasli, S. W. Miller, R. Mozzi, and L. K. Hanes, "Capacitively coupled traveling-wave power amplifier," IEEE Trans. Electron. Devices, vol. 31, pp. 1937-1942, Dec. 1984.
[4] Y. Ayasli, L. D. Reynolds, R. L. Mozzi, and L. K. Hanes, "2-20-GHz GaAs traveling-wave power amplifier," IEEE Trans. Microwave Theory Tech., vol. 32, pp. 290-295, Mar. 1984.

[5] R. Halladay, M. Jones, and S. Nelson, "A producible 2 to 20-GHz monolithic power amplifier," in IEEE 1987 Microwave and Millimeter-Wave Monolithic Circuits Symp. Dig., C. Huang, Ed., 1987, pp. 19-21.

[6] M. Campovecchio, B. Le Bras, M. Lajugie, and J. Obregon, "Optimum design of distributed power-FET amplifiers. Application to a $2-18-\mathrm{GHz}$ MMIC module exhibiting improved power performances," in IEEE 1994 Microwave and Millimeter-Wave Monolithic Circuits Symp. Dig., L. Liu, Ed., 1993, pp. 125-128.

[7] K. Krishnamurthy, S. I. Long, and M. J. W. Rodwell, "Cascode-delaymatched distributed amplifiers for efficient broadband microwave power amplification," in IEEE 1999 Microwave and Millimeter-Wave Monolithic Circuits Symp. Dig., M. Matloubian and E. Ponti, Eds., 1999, pp. 819-822.

[8] K. Krishnamurthy, M. J. W. Rodwell, and S. I. Long, "A > 25\% PAE 0.2-6-GHz lumped power amplifier in a 18-GHz MESFET technology," in IEEE 1999 GaAs IC Symp. Dig., Oct. 1999, pp. 81-84.

[9] Tektronix Inc., Beaverton, OR, "Monolithic Wideband Amplifier," US Patent No. 4236 119, Nov. 25, 1980.

[10] B. W. Lai, "Amplification beyond $f t$ using non-inductive circuit techniques," M.S. thesis, Dept. Elect. Eng. Comput. Sci., Univ. California, Berkeley, CA, May 1983.

[11] C. T. Armijo and R. G. Meyer, "A new wideband Darlington amplifier," IEEE J. Solid-State Circuits, vol. 24, pp. 1105-1109, Apr. 1989.

[12] Y.-F. Wu, B. P. Keller, S. Keller, N. X. Nguyen, M. Le, C. Nguyen, T. J. Jenkins, L. T. Kehias, S. P. Denbaars, and U. K. Mishra, "Short channel AlGaN/GaN MODFETs with $50-\mathrm{GHz} f_{\tau}$ and $1.7 \mathrm{~W} / \mathrm{mm}$ output power at $10 \mathrm{GHz}, "$ IEEE Electron. Device Lett., vol. 18, no. 9, pp. 438-440, 1997.

[13] K. Krishnamurthy, R. Vetury, S. Keller, M. J. W. Rodwell, S. I. Long, and U. Mishra, "Broadband microwave power amplifiers in GaN technology," in IEEE Topical Workshop on Power Amplifiers for Wireless Communications Dig., San Diego, CA, Sept. 13-14, 1999, pp. 20-21.

[14] S. C. Cripps, "A theory for the prediction of GaAs FET load-pull power contours," in IEEE 1983 Microwave and Millimeter-Wave Monolithic Circuits Symp. Dig., pp. 221-223.

[15] B. J. Thibeault, B. P. Keller, P. Fini, U. K. Mishra, C. Nguyen, N. X. Nguyen, and M. Le, "High performance and large area flip-chip bonded AlGaN/GaN MODFETs," in Int. Electron. Devices Meeting Tech. Dig., 1997, pp. 569-572.

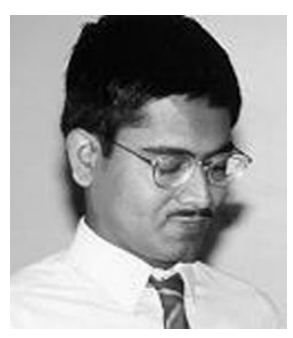

Karthikeyan Krishnamurthy received the B.Tech. degree from the Indian Institute of Technology, Chennai, in 1995, and the M.S. and Ph.D. degrees from the University of California, Santa Barbara (UCSB), in 1996 and 2000, respectively.

$\mathrm{He}$ is currently a Senior Engineer at Gtran, Inc., Westlake, CA. His research at UCSB focused on circuits for ultra-broadband efficient high-power microwave amplifiers in AlGaN/GaN HEMT technology.

$\mathrm{He}$ is a recipient of the Delco Defense Systems Operations Graduate Fellowship and was awarded the second best student paper award at the 1999 International Microwave Symposium for his work on efficient distributed power amplifiers.

Ramakrishna Vetury, photograph and biography not available at time of publication

Stacia Keller, photograph and biography not available at time of publication. 
Umesh Mishra received the Ph.D. degree from Cornell University, Ithaca, NY, in 1984.

$\mathrm{He}$ is a Professor in the Electrical and Computer Engineering Department, University of California, Santa Barbara, and a Cofounder of Widegap Technology LLC, Goleta, CA. His current research interests are in oxide-based III-V electronics and III-Nitride electronics and optoelectronics. He is recognized as one of the leaders in the development of AlInAs-GaInAs HEMTs and HBTs from research into reliable product. He has received five patents and has nine pending. He has authored or co-authored more than 200 papers in technical journals and conferences and has delivered more than 50 invited lectures at conferences.

Dr. Mishra received the Young Scientist of the Year Award from the International Symposium on GaAs and Related Compounds. He also received a Hyland Patent Award, which is presented by Hughes Aircraft.

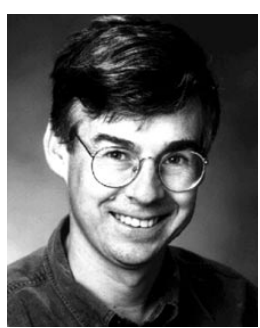

Mark J. W. Rodwell received the B.S. degree from the University of Tennessee, Knoxville, in 1980, and the M.S. and Ph.D. degrees from Stanford University, Stanford, CA, in 1982 and 1988, respectively.

$\mathrm{He}$ is a Professor and Director of the Compound Semiconductor Research Laboratories, University of California, Santa Barbara. He was with AT\&T Bell Labs during 1982-1984. His research focuses on very high-bandwidth bipolar transistors, high-speed bipolar IC design, and gigahertz mixed-signal ICs. His group has worked extensively in the area of GaAs Schottky-diode ICs for subpicosecond/millimeter-wave instrumentation.

Dr. Rodwell received a 1989 National Science Foundation Presidential Young Investigator Award. His work on submillimeter-wave diode ICs was awarded the 1997 IEEE Microwave Prize.

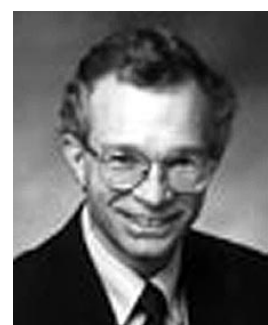

Stephen I. Long received the B.S. degree in engineering physics from the University of California, Berkeley, in 1967, and the M.S. and Ph.D. degrees in electrical engineering from Cornell University, Ithaca, NY, in 1969 and 1974, respectively.

From 1974 to 1977, he was a Senior Engineer and Manager of Semiconductor Engineering at Varian Associates, Palo Alto, CA. From 1978 to 1981 , he was a Member of Technical Staff at Rockwell International Science Center, Thousand Oaks, CA. In 1981, he joined the Electrical and Computer Engineering Department, University of California, Santa Barbara, where he is currently a Professor. His research interests include the design of low-power high-speed digital and analog circuits, high-performance devices and fabrication technologies, and microwave analog integrated circuits for personal communications. In 1988, he was a Research Visitor at GEC Hirst Research Centre, U.K. In 1994, he was a Fulbright Research Visitor at the Signal Processing Laboratory, Tampere University of Technology, Finland, and a Visiting Professor at the Electromagnetics Institute, Technical University of Denmark. In 1999, he was a Visitor at the Hewlett-Packard HP/EE division, Santa Rosa, CA

Dr. Long received the IEEE Microwave Applications Award in 1978 for development of $\mathrm{InP}$ millimeter-wave devices. 